\title{
A Study on the Impact of Organizational Identity on the new generation of migrant workers' Turnover intention: a mediated model of mediation
}

\author{
Xiupeng Ding ${ }^{a}$, Lu Ma ${ }^{b}$ \\ School of Management, Guangxi University of Science and Technology, Liuzhou 545006, China \\ a65438284@qq.com, bmalu6655@163.com
}

Keywords: Organizational Identity, Turnover intention, the new generation of migrant workers, intrinsic motivation.

\begin{abstract}
Through the method of literature research, it is found that organizational identity and job embedding are paid more and more attention by satisfying the needs of the new generation of migrant workers' career development and social psychological needs. Based on the theory of work motivation, this paper discusses the relationship between organizational identity and the wishes of the new generation of migrant workers and the mechanism of action. The results show that: (1) organizational identity is negatively correlated with the willingness of the new generation of migrant workers; (2) the work is embedded in the organizational recognition and the new generation of migrant workers from the job intention to play a mediating role; (3) internal work motivation to adjust the organization, Negative relationship between migrant workers' turnover intention. And on this basis, enterprise*s should actively carry out and improve the human resources management projects.
\end{abstract}

\section{Introduction}

According to the official statistics of the state, the number of migrant workers in China in 2016 has reached 280 million, of which more than $49.7 \%$ were born after 1980 . With the accelerated pace of urbanization in China, more and more young generation to leave the countryside to enter the city to work life, so the new generation of migrant workers gradually replace the older generation of migrant workers to become the main body of urban construction. But migrant workers are often accompanied by a high turnover rate because of their job instability. Wang Bangjun (2014) study shows that the frequent turnover of the new generation of migrant workers not only to the enterprise to bring the risk of production technology loss, but also increase the cost of corporate recruitment and training, affecting the production efficiency and competitiveness of enterprises [1]. Sun Zhongwei and Yang Xiaofeng (2012) found that because the new generation of migrant workers choose to work, often refer to the flow of people around the work or career, resulting in frequent turnover and job-hopping, and to some enterprises to bring "migrant workers" phenomenon [2]. With the older generation of migrant workers, "working to survive," the reality of the dilemma of the new generation of migrant workers growth experience, self-psychological orientation, the city's motivation and the main demands have changed significantly. They are pickier for pay, working conditions, living environment, and their job stability and loyalty are also significantly weakened. Therefore, how to reduce the turnover rate of the new generation of migrant workers, and enhance their efficiency is an important problem that enterprise managers need to solve.

At present, some scholars at home and abroad have done some theoretical and empirical research on the relationship between organizational identity, job embedding and the wishes of the new generation of migrant workers, but the boundary condition of the mechanism between them is not clear. Therefore, this paper chooses to explore and study the mechanism of organizational identity, job embedding and turnover intention from the perspective of the inherent motivation of the new generation of migrant workers. The ultimate goal is to provide theoretical guidance for the decision-making of the organization's managers and to provide rationalization proposals to reduce the turnover rate of the new generation of migrant workers. 


\section{Main research contents}

\subsection{The relationship between organizational identity and the wishes of the new generation of migrant workers}

The organizational identity of the individual influences the employee's psychology and ultimately affects performance through the employee's behavioral response. Employee if the organization identify very weave and in action on the side of the organization, and then he'll consciousness or subconsciously psychologically attached to groups such as they may think to give them treatment is reasonable and fair, because they are hard to the temptation of the outside world and leave the organization. Dick (2005) found that individuals with high levels of identification can improve their job satisfaction ${ }^{[3]}$. Research by people such as Bamber (2002) shows that organizational identification can significantly reduce professional conflict in the organization, significantly reducing the individual's intention to leave the office by ${ }^{[5]}$. The role of the new generation of migrant workers from its dual in farmers and the urban workers identity cognition orientation of role conflict on cognitive orientation, and the essence of the conflict is also constitute a new generation of migrant workers are the important factors of organizational identification, Zhong Chuanxin (2012) the employee organizational identification would impact on the psychological and behavior of members of the organization: organization highly esteemed employees tend to see themselves as the representative of the organization, the collective interests as their primary factor ${ }^{[6]}$.

Therefore, can be found: the new generation of migrant workers group identity affects their organizational behavior, when organizations were high, they have a high degree of satisfaction and the characteristics of low turnover intention, prone to organizational citizenship behavior, and hold high manner of business cooperation, personal behavior, decision making, and activity are more likely to be in line with the interests of the organization.

\subsection{The relationship between job embedding and the wishes of the new generation of migrant workers}

Past studies have shown that individual job embedding can significantly affect its performance in the organization. When individuals are deeply embedded in the organization, they will establish a deep connection with the organization and feel very suitable for development within the organization. Once they choose to leave, they will lose the established interpersonal network and the identity of the original organization status. So the high degree of embedded individuals are often high in the organization of performance. The study of Chan (1996) shows that individual job embedding is negatively correlated with turnover intention ${ }^{[7]}$. Lee (2004) argues that job embedding is related to individual turnover negative, and job embedding is positively related to individual performance and organizational citizenship behavior. The new generation of migrant workers' work embedded model has a special side ${ }^{[8]}$. Jia Wenjuan (2006) suggested that in migrant workers more enterprises, there are generally "foremen", these foremen master a lot of information and labor market in the middle of the intermediary position, their own affinity, geography will affect the Other migrant workers' job embedding ${ }^{[9]}$. Qingping (2012) through empirical research found that the embedded work within the job has a significant negative impact ${ }^{[10]}$. Sun Zhongwei (2012) further confirmed that the theory of job embedding has played a negative role in the turnover intention of the new generation of migrant workers ${ }^{[2]}$.

Thus, it can be found that when the new generation of migrant workers embedded in the negative impact on their turnover intention. The higher the job embedding, the better the match between the employee and the enterprise, the more positive attitude and the action of the enterprise project, and the employee to show more job performance and reduce the turnover tendency.

\subsection{The Relationship between Organizational Identity and the Embedding of New Generation Peasant Workers}

Mitchell (2001) et al. Proposed the idea that the embedded embedding variables are considered to have the same intermediate status as the work attitude variables to study the departure model ${ }^{[4]}$. Chinese scholar Liang Xiaowei, Liao Jianqiao (2005) introduced a model of resignation / retention, and expanded and examined Mitchell et al.'s vision in subsequent studies ${ }^{[11]}$. Allen, David G (2006), 
through a new employee survey of a large financial services organization, found that social adaptation techniques enable new employees to be actively embedded in the organization, where collective, fixed, and authorized skills are embedded Significant positive correlation ${ }^{[12]}$. Yuan Qinghong and Chen Wenchun (2008) for the organization's awareness, motivation and organization of culture, human resources practices are likely to affect the individual's work embedded ${ }^{[13]}$.

Therefore, on this basis, we can find that the new generation of migrant workers organizational identity has a positive impact on job embedding. The higher the degree of organizational recognition, the higher the degree of employee recognition for the enterprise, so that the higher the embedded work, the staff will have a higher efficiency.

\subsection{The relationship between the new generation of migrant workers' work embedded in organizational recognition and turnover intention}

Organizational identity is an individual self-perceived process and the resulting psychological attribution, belonging to the "knowledge" level of work is embedded in the individual as a result of various factors embedded in the organization of a degree, are "line" Level. In accordance with the usual logic of cognitive behavior, "knowledge" should be in the former, "line" should be in the future, only when people have a certain understanding of things, will show the corresponding behavior. Therefore, we believe that it is logically logical to combine organizational identity as an adversary variable embedded in work. And we often see in the life of the organization has a high degree of recognition of the staff of the high degree of embedded work on the organization of low degree of staff work embedded low, but in turn, when we see some employees work embedded Very high, it may be a lot of complaints about the organization, such as some managers because of the salary and the superiority of the post so that although they are not suited to the organization's culture, but also chose to stay in the organization to continue the service behavior. Qin Weiping (2014) found that improving the organizational identity of the new generation of migrant workers can effectively improve their job embedding ${ }^{[14]}$. Wang Zhanjun (2014) shows that the matching dimension of the new generation of migrant workers' embedded work plays an intermediary role in the influence of organizational identity on job performance ${ }^{[1]}$.

Therefore, there is a significant positive correlation between organizational identity and job embedding, and there is a significant negative correlation with job intention. There is a significant negative correlation between job embedding and job intention. Job embedding has a complete mediating role in organizational recognition and turnover intention.

\subsection{The adjustment of the intrinsic motivation of the new generation of migrant workers}

For the identity of the new generation of migrant workers, many scholars found that the new generation of migrant workers and their parents are different, their identity is vaguer. Peng Yuanchun (2007) in the study of migrant workers in Wuhan, found that wage income is not the impact of migrant workers identity factors, and monthly income satisfaction on the identity of the new generation of migrant workers have a significant impact [15] . Li Yibo (2010) that the new generation of migrant workers within the group of self-identity differentiation, some new generation of migrant workers will be self-identity as farmers, some people think they belong to the public [16]. When the new generation of migrant workers enter the city, the reference groups in terms of income and status are inevitably diverted from urban peasants with high homogeneity to urban residents, resulting in unfairness and relative deprivation. So their motivation to change into the city also changed from the initial in order to make money to support their families, such as home subsidies and other economic motives have been transformed into the pursuit of equality, desire to integrate into the city, treat the work also have career expectations. Yao Shifu(2012) and others have shown that when the new generation of migrant workers have the desire to work and career development, the wages of enterprises cannot obviously affect their job satisfaction, they are more pursuit of fair treatment, job promotion And the development of personal values [17].

Therefore, the internal motivation of the work can regulate the new generation of migrant workers organizational identity, the new generation of migrant workers organization high degree of their work embedded in the higher, the lower the willingness to leave. 


\section{Summary}

\subsection{Main Conclusions}

On the basis of previous studies, it is found that: (1) Organizational identity will affect the turnover intention of the new generation of migrant workers; (2) New generation of migrant workers' work has a negative impact on the intention to leave; (3) The new generation of migrant workers' organizational identity has a positive impact on job embedding ; (4) new generation of migrant workers embedded in organizational recognition and turnover between the desire to have a mediating role.( 5) new generation of migrant workers in the work of the positive adjustment of organizational recognition of the intention to leave the job The indirect effect of the effect. Therefore, the new generation of migrant workers organization recognition, job embedding, intrinsic motivation and its role in the mechanism of turnover, can be used in Figure 1.

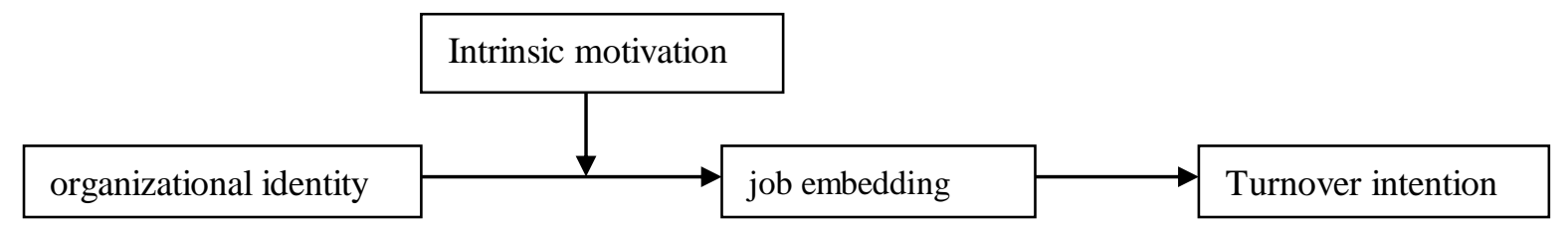

Figure 1. Theoretical model figure

\subsection{Countermeasures and Suggestions}

(1) When recruiting new generations of migrant workers, they should fully consider their degree of recognition of corporate culture and values, and recruit employees with high similarity to organizational values. (2) in the management of the new generation of migrant workers, should be targeted to human management, for those who want to stay in the city staff, you can give full consideration to the individual needs of employees to strengthen their ability to cultivate their career planning, Their sense of belonging(3) managers to fully understand their work trends, such as the organization that employees can reduce the organization, but also through communication channels, the use of organizational support, leadership and other means, so that employees feel the organization's emotional attention and concern, Its ability to trust, so as to stimulate the staff of the organization's identity, deeply feel that they are members of the organization, in order to be able to work in the organization and proud. Such organizational identity is often positively related to positive work attitude and behavioral variables, such as enhancing organizational loyalty and improving job performance; negatively related to negative work attitude and behavioral variables, such as lower turnover rates, etc., making them better embedded Organize, inspire their organizational citizenship behavior and improve job performance.

\section{Acknowledgements}

Fund project: Innovation Project of GuangXi University of Science and Technology Graduate Education (GKYC201612)

\section{References}

[1] Wang Bangjun, Yang Dongtao. Cenozoic migrant workers' organization identity work and its workEmpirical Study on the Impact of Performance [J] Soft Science, 2014,28 (1): 106-109.

[2] Sun Zhongwei, Yang Xiaofeng. Dehased employment relationship and willingness of migrant workers to leave - based on the questionnaire survey in the Yangtze River Delta and the Pearl River Delta [J]. Society, 2012 (3): 98-128.

[3] Riketta M, Van Dick, R Foci. Attachment in Organizations: AMeta - analytic comparison of the Strength and Correlates of Work-group Versus Organizational Identification and Commitment [J]. Journal of Vocational Behavior, 2005, (67): 490.

[4] Mitchell T R, Holtom B C, Lee T W, et al. Hyuse Stay: UsingJob Embeddedness to Predict Voluntary Turnover [J]. Academy ofManagement Journal, 2001, 44 (6): 1102-1121. 
[5] Bamber EM, Iyer V M.Big5AuditorsProfessionaland Organizational Identification: Consistency or Conflict? [J]. Auditing: A Journal of Punice \& Theory, 2002, 21 (2): 21-38.

[6] Zhong Chuanxin. Organization identity, job embedding and its impact on job performance [J]. China Management Information, 2012, (08): 73-75.

[7] Chan D. Cognitive Misfit of Problem Solving Style at Word: A Facet of Person -Organization Fit [J]. Organizational Behavior and Human Decision Processes, 1996, 68 (3): 194-207.

[8] Lee T W, Terence R, Mitchell Chris J. Et al. The Effects of Job Embeddedness on Organizational Citizenship, Job Performance, Volitional Absences, and Voluntary Turnover [J]. Academy of Management Journal, 2004, 47 (5), 711-722.

[9] Jia Wenjuan "foreman" power source and its impact on labor relations a historical comparison of perspective [J]. Society, 2006 (5): 134-157

[10] Qing ping, zidane, Nie Ping. Embedded work of college students "village official" departure will study - on work values as regulating variable [J]. Agricultural technology economy, 2012 (04) : 14 $-23$

[11] Liang Xiaowei, Liao Jianqiao, Zeng Qinghai. Based on the work of embedded core staff organizational performance --- voluntary turnover research model expansion and testing [J]. Managing the World, 2005 (7): 106-115.

[12] David G Allen. Do Organizational Socialization Tactics InfluenceNewcomer Embeddedness and Turnover? [J]. Journal of Management, 2006, 32 (2): 237-256.

[13] Yuanqing Hong, Chen Wenchun. Work embedded concepts, measurements and related variables [J]. Advances in Psychological Science, 2008, 16 (6): 941.[J]. Journal of Management, 2014, (10): 1445-1452.

[14] Qin Weiping, Zhao Shuming.Study on the Relationship between Organizational Justice and Job Embedding in the New Generation of Migrant Workers

[15] Peng Yuanchun. On the Identity of Migrant Workers and Its Influencing Factors - An Investigation and Analysis of the Catering Workers in Yangyuan Community in Wuhan [J]. Population Research, 2007, (02): 81-90.

[16] Li Yibo, Zhu Kaojin. Constitution of migrant workers subjective cognition: identity, urban ownership and development planning - Taking 1085 migrant workers in Jiangsu Province as an example [J]. Journal of Nanjing Agricultural University (Social Science Edition), 2010, (01): 96-101.

[17] Yao Zhifu, Zhang Yiwen. Analysis of Factors Affecting Job Satisfaction of Migrant Workers in New Generation - Based on the Survey Data of Four Provinces in Northwest China [J]. China Rural Economy .2012, (8): 46-55.

\section{Author in brief:}

Ding xiupeng, postgraduate studying in Guangxi University of science and Technology, mainly engaged in Organizational behaviour and Human resource management.E-mail:654382841@qq.com 